This item was submitted to Loughborough's Research Repository by the author.

Items in Figshare are protected by copyright, with all rights reserved, unless otherwise indicated.

\title{
Singular and regular gap solitons between three dispersion curves
}

PLEASE CITE THE PUBLISHED VERSION

PUBLISHER

(C) The American Physical Society

LICENCE

CC BY-NC-ND 4.0

\section{REPOSITORY RECORD}

Grimshaw, Roger H.J., Boris A. Malomed, and Georg A. Gottwald. 2019. "Singular and Regular Gap Solitons Between Three Dispersion Curves”. figshare. https://hdl.handle.net/2134/709. 
Cuspons, peakons and regular gap solitons between three dispersion curves

\author{
Roger Grimshaw ${ }^{1}$ \\ Department of Mathematical Sciences, Loughborough University, \\ Loughborough, LE11 3TU, UK \\ Boris A. Malomed ${ }^{2}$ \\ Department of Interdisciplinary Studies, Faculty of Engineering, \\ Tel Aviv University, Tel Aviv 69978, Israel
}

Georg A. Gottwald ${ }^{3}$

Department of Mathematics and Statistics, Surrey University, Guildford, GU2 7XH, UK

\footnotetext{
${ }^{1}$ e-mail R.H.J.Grimshaw@lboro.ac.uk

2e-mail malomed@eng.tau.ac.il

${ }^{3}$ e-mail g.gottwald@surrey.ac.uk
} 


\begin{abstract}
A general model is introduced which describes a system with cubic nonlinearities, in a situation when the linear dispersion relation has three branches which nearly intersect. The system includes two waves with a strong linear coupling between them, to which a third wave is coupled. This model has two gaps in its linear spectrum. A nonlinear analysis is performed for zero-velocity solitons. If one may disregard the self-phase modulation (SPM) term in the equation for the third wave, we find an analytical solution which shows that there simultaneously exist two different families of generic solitons: regular ones, which may be regarded as a smooth deformation of the usual gap solitons in the two-wave system, and cuspons, which have finite amplitude and energy, but a singularity in the first derivative at their center. Even in the limit when the linear coupling of the third wave to the first two nearly vanishes, the soliton family remains drastically different from that in the uncoupled system: regular solitons whose amplitude exceeds a critical value are replaced in this limit by peakons. While the regular solitons, cuspons, and peakons are found in an exact analytical form, their stability is tested numerically, which shows that they all may be stable. If the SPM terms are retained, we find that there again simultaneously exist two different families of generic stable soliton solutions, viz., regular ones and peakons, whose existence depends on the sign of certain system parameters. Direct simulations show that both types of the solitons may be stable in this case too.
\end{abstract}

PACS numbers: $05.45 . \mathrm{Yv}$; 42.65.Tg; 42.81.Dp; 47.55.Hd 


\section{Introduction}

\subsection{The model system and solitons}

Gap solitons (GS) is a common name for solitary waves in nonlinear models which feature one or more gaps in their linear spectrum [1]. A soliton may exist if its frequency belongs to the gap, as then it does not decay into linear waves.

Gaps in the linear spectrum are a generic phenomenon in two- or multicomponent systems, as intersection of dispersion curves belonging to different components is generically prevented by a linear coupling between the components. Excluding cases when the zero solution in the system is unstable [2], the intersection avoidance alters the spectrum so that a gap opens in place of the intersection. Approximating the two dispersion curves, that would intersect in the absence of the coupling, by straight lines, and assuming a generic cubic nonlinearity, one arrives at a generalized massive Thirring model (GMTM), which has a family of exact GS solutions that completely fill the gap [3]. The model has a direct application to nonlinear optics, describing co-propagation of left- and right-traveling electromagnetic waves in a fiber with a resonant Bragg grating (BG). Gap solitons, first predicted theoretically, were observed in experiments with light pulses launched into a short piece of the BG-equipped fiber [4] (in fact, optical solitons that were first observed in the BG fiber [5] were, strictly speaking, not of the GS type, but more general ones, whose central frequency did not belong to the fiber's bandgap).

GSs are known not only in optics but also in other physical situations, for instance, in density-stratified fluid flows, where dispersion curves pertaining to different internal-wave modes can readily intersect. Taking into regard the nonlinearity, one can easily predict the occurrence of GS in density-stratified fluids [6].

In this work, we aim to consider GS that may exist in a situation when the underlying system contains three wave components, and there is a parametric region in which the corresponding three dispersion curves are close to intersection at a single point, unless linear couplings are taken into regard. Of course, the situation with three curves passing through a single point is degenerate. Our objective is to investigate GS not for this special case, but in its vicinity in the parameter space.

Situations of this type can be readily occur in the above-mentioned density-stratified flows [7], and are also possible in optics. For instance, this case takes place in a resonantly absorbing BG, which are 
arranged as a system of thin $(\sim 100 \mathrm{~nm})$ parallel layers of two-level atoms, with the spacing between them equal to half the wavelength of light. This system combines the resonant Bragg reflection and self-induced transparency (SIT), see Ref. [8] and references therein. A model describing the BG-SIT system includes equations for three essential fields, viz., local amplitudes of right- and left-traveling electromagnetic waves, and the inversion rate of the two-level atoms (which, obviously, has zero group velocity in the laboratory reference frame). This model indeed produces a linear spectrum with three dispersion curves close to intersecting at one point, so that two gaps open in the system's spectrum.

Another realization of gaps between three dispersion curves is possible in terms of stationary optical fields in a planar nonlinear waveguide equipped with BG in the form of parallel scores [9]. In this case, the resonant Bragg reflection linearly couples waves propagating in two different directions. To induce linear couplings between all the three waves in the system, it is necessary to have a planar waveguide with two different BG systems of parallel scores, oriented in different directions. Postponing a consideration of this rather complicated model to another work, we here give a simple example for a case when the single BG is aligned along the axis $x$, perpendicular to the propagation direction $z$. Two waves $u_{1,2}$ have opposite incidence angles with respect to the $\mathrm{BG}$, while the third wave $u_{3}$ has its wave vector parallel to $x$, see Fig. 1 in Ref. [9]. Then, assuming that the size of the sample is much smaller than the diffraction length of a broad spatial beam, but is larger than a characteristic length induced by strong artificial diffraction induced by BG, normalized equations governing the spatial evolution of the fields in the planar waveguide with the usual Kerr nonlinearity (unlike the second-harmonic-generating nonlinearity considered in Ref. [9]) are

$$
\begin{gathered}
i\left(\frac{\partial u_{1}}{\partial z}-\frac{\partial u_{1}}{\partial x}\right)+u_{2}+\left(\frac{1}{2}\left|u_{1}\right|^{2}+\left|u_{2}\right|^{2}+\left|u_{3}\right|^{2}\right) u_{1}=0 \\
i\left(\frac{\partial u_{2}}{\partial z}+\frac{\partial u_{2}}{\partial x}\right)+u_{1}+\left(\frac{1}{2}\left|u_{2}\right|^{2}+\left|u_{1}\right|^{2}+\left|u_{3}\right|^{2}\right) u_{2}=0 \\
i \frac{\partial u_{3}}{\partial z}+\left(\frac{1}{2}\left|u_{3}\right|^{2}+\left|u_{1}\right|^{2}+\left|u_{2}\right|^{2}\right) u_{3}=k_{0} u_{3},
\end{gathered}
$$

where $k_{0}$ is a wavenumber mismatch between the third and first two waves.

The model based on Eqs. (1) - (3) represents a particular case only, as it does not include linear couplings between the waves $u_{1,2}$ and $u_{3}$. We aim to introduce a generic model describing a nonlinear system of three waves with linear couplings between all of them. We assume that the system can be derived from a Hamiltonian, and confine attention to the case of cubic nonlinearities. Taking into regard 
these restrictions, and making use of scaling invariances to diminish the number of free parameters, we arrive at a system

$$
\begin{gathered}
i\left(\frac{\partial u_{1}}{\partial t}-\frac{\partial u_{1}}{\partial x}\right)+u_{2}+\kappa u_{3}+\alpha\left(\alpha \sigma_{1}\left|u_{1}\right|^{2}+\alpha\left|u_{2}\right|^{2}+\left|u_{3}\right|^{2}\right) u_{1}=0 \\
i\left(\frac{\partial u_{2}}{\partial t}+\frac{\partial u_{2}}{\partial x}\right)+u_{1}+\kappa u_{3}+\alpha\left(\alpha \sigma_{1}\left|u_{2}\right|^{2}+\alpha\left|u_{1}\right|^{2}+\left|u_{3}\right|^{2}\right) u_{2}=0 \\
i \frac{\partial u_{3}}{\partial t}+\kappa\left(u_{1}+u_{2}\right)+\left(\sigma_{3}\left|u_{3}\right|^{2}+\alpha\left|u_{1}\right|^{2}+\alpha\left|u_{2}\right|^{2}\right) u_{3}=\omega_{0} u_{3} .
\end{gathered}
$$

Here, we consider the evolution in the temporal domain, unlike the spatial-domain evolution in Eqs. (1) - (3), and without loss of generality, we use a reference frame in which the third wave $u_{3}$ has zero group velocity. We also assume full symmetry between the two waves $u_{1,2}$, following the pattern of the GMT model; in particular, the group velocities of these waves are normalized to be $\mp 1$. However, we note that this assumption is not essential, and we shall comment later on the case when the group-velocity terms in Eqs. (4) and (5) are generalized as follows:

$$
-\frac{\partial u_{1}}{\partial x} \rightarrow-c_{1} \frac{\partial u_{1}}{\partial x},+\frac{\partial u_{2}}{\partial x} \rightarrow+c_{2} \frac{\partial u_{1}}{\partial x},
$$

where $c_{1}$ and $c_{2}$ are different, but have the same sign (positive say).

In the case when Eqs. (4) - (6) apply to density-stratified flows, or to the description of the spatial evolution of the electromagnetic fields in a planar nonlinear waveguide equipped with BG (cf. Eqs. (1) (3)), the system with the full symmetry between the fields $u_{1}$ and $u_{2}$ represents, indeed, a particular case. However, in the optical models of the BG-SIT type (in the temporal domain), when the variables $u_{1,2}$ are amplitudes of two counterpropagating waves in the fiber, while $u_{3}$ is an immobile material variable like the population inversion, the symmetry assumed in Eqs. (4) - (6) is generic. Note that the coefficient of the linear coupling between the first two waves is normalized to be 1 , while $\kappa$ accounts for their linear coupling to the third wave, and it may always be defined to be positive.

We also assume the natural symmetry of the system's dispersion law $\omega=\omega(k)$ with respect to the sign of $k$, but not of $\omega$. To this end, we have the parameter $\omega_{0}$ in Eq. (3) which breaks the " $\omega$-symmetry", that, unlike the " $k$-symmetry", does not have any natural cause to exist.

The coefficients $\sigma_{1,3}$ and $\alpha$ in Eqs. (4) - (6) account for the nonlinear self-phase modulation (SPM) and cross-phase modulation (XPM), respectively. In particular, $\alpha$ is defined as a relative XPM coefficient between the first two and the third waves, hence it is an irreduceable parameter. As for the SPM 
coefficients, both may be normalized to be \pm 1 , unless they are equal to zero; however, it will be convenient to keep them as free parameters, see below (note that the SPM coefficients are always positive in the optical models, but in those describing stratified fluids they may have either sign). Normalizations of the nonlinear terms different from that adopted in Eqs. (4) - (6 ) are possible too, but the number of the parameters will be the same. We keep only the most natural nonlinear SPM and XPM terms in Eqs. (4) - (6) (the terms of the same types as in the standard GMT model). In particular, possible nonlinear corrections to the linear couplings (for instance, a term $\sim\left|u_{1}\right|^{2} u_{2}$ in Eq. (4)) are known to be negligible in models of optical media.

Equations (4) - (6) conserve the norm, which is frequently called energy in optics,

$$
N \equiv \sum_{n=1,2,3} \int_{-\infty}^{+\infty}\left|u_{n}(x)\right|^{2} d x
$$

the Hamiltonian,

$$
\begin{aligned}
H & \equiv H_{\text {grad }}+H_{\text {coupl }}+H_{\text {focus }} \\
H_{\text {grad }} & \equiv \frac{i}{2} \int_{-\infty}^{+\infty}\left(u_{1}^{*} \frac{\partial u_{1}}{\partial x}-u_{2}^{*} \frac{\partial u_{2}}{\partial x}\right) d x+\text { c.c. } \\
H_{\text {coupl }} & \equiv-\int_{-\infty}^{+\infty}\left[u_{1}^{*} u_{2}+\kappa u_{3}^{*}\left(u_{1}+u_{2}\right)\right] d x+\text { c.c. } \\
H_{\text {focus }} & \equiv-\int_{-\infty}^{+\infty}\left[\frac{1}{2} \alpha^{2} \sigma_{1}\left(\left|u_{1}\right|^{4}+\left|u_{2}\right|^{4}\right)+\frac{1}{2} \sigma_{3}\left|u_{3}\right|^{4}+\alpha^{2}\left|u_{1}\right|^{2}\left|u_{2}\right|^{2}+\alpha\left|u_{3}\right|^{2}\left(\left|u_{1}\right|^{2}+\left|u_{2}\right|^{2}\right)\right] d x
\end{aligned}
$$

and the momentum, which will not be used here. In these expressions, the asterisk and c.c. both stand for complex conjugation, $H_{\text {grad }}, H_{\text {coupl }}$, and $H_{\text {focus }}$ being the gradient, linear-coupling, and self-focusing parts of the Hamiltonian.

Our objective is to find various types of solitons existing in the generic three-wave system (4) - (6) and investigate their stability. Focusing first on the case (suggested by the analogy with GMTM) when the SPM term in Eq. (6) may be neglected (i.e., $\sigma_{3}=0$ ), in section 2 we find a general family of zero-velocity solitons in an exact analytical form. We will demonstrate that they are of two drastically different types: regular GSs, and cuspons, i.e., solitons with a cusp singularity at the center; however, the amplitude and energy of the cuspons are finite. Cuspons are known to exist in degenerate models without linear terms (except for the evolution term such as $\partial u / \partial t$ ), i.e., without a linear spectrum, a well-known example being the exactly integrable Camassa-Holm $(\mathrm{CH})$ equation $[13,14]$. Our model resembles the $\mathrm{CH}$ one 
in the sense that both give rise to coexisting solutions in the form of regular solitons and cuspons. The cause for the existence of these singular solitons in our model is the fact that, looking for a zero-velocity soliton solution, one may eliminate the field $u_{3}$ by means of an algebraic relation following, in this case, from Eq. (6). The subsequent substitution of this result into the first two equations (4) and (5) produces a non-polynomial (in fact, rational) nonlinearity in them. The corresponding rational functions feature a singularity at some (critical) value of the soliton's amplitude. If the amplitude of a formal regular-soliton solution exceeds the critical value, it actually cannot exist, and in the case when $\sigma_{3}=0$ it is replaced by a cuspon, whose amplitude is exactly equal to the critical value.

In the limit $\kappa \rightarrow 0$, which corresponds to the vanishing linear coupling between the first two and third waves, the cuspon resembles a peakon, which is a finite-amplitude solitary wave with a jump of its first derivative at the center. Note that peakon solutions, coexisting with regular solitons (this property is shared by our model), are known in a slightly different (also integrable) version of the $\mathrm{CH}$ equation, see, e.g., Ref. $[13,15,16]$.

Then, we show that, when the SPM term in Eq. (6) is restored in Eq. (6) (i.e., $\sigma_{3} \neq 0$; the presence or absence of the SPM term $\propto \sigma_{1}$ in Eqs. (4) and (5) is not crucially important), the system supports a different set of soliton families. These are regular GSs and, depending on the sign of certain parameters, a family of peakons, which, this time, appear as generic solutions, unlike the case $\sigma_{3}=0$, when they only exist as a limiting form of the solutions corresponding to $\kappa \rightarrow 0$. As far as we know, the model formulated in the present work is the first non-degenerate one (i.e., a model with a nonvanishing linear part) which yields both cuspons and peakons.

\subsection{Stability of the solitons and spatiotemporal collapse}

As concerns the dynamical stability of the various solitons in the model (4)-(6), in this work we limit ourselves to direct numerical simulations, as a more rigorous approach, based on numerical analysis of the corresponding linear stability-eigenvalue problem [17], is technically difficult in the case of cuspons and peakons (results of such an analysis, based on the Evans-function technique, will be presented elsewhere). In fact, direct simulations of perturbed cuspons and peakons is a hard problem too, but we have concluded that identical conclusions concerning the stability have been obtained (see section 3 below) by means of high-accuracy finite-difference and pseudo-spectral methods (each being implemented in more than one 
particular form), which lends the results credibility. A general conclusion is that the regular solitons are always stable, although when they occur for parameter values close to the parameter border for cuspons, they exhibit internal vibrations. As for the cuspons and peakons, they may be either stable or unstable. If the cusp is strong enough, instability of the cuspon initiates formation of a genuine singularity, i.e., onset of a spatiotemporal collapse [18] in the present one-dimensional model.

Note that a simple virial-type estimate for the possibility of collapse can be done, assuming that the field focuses itself in a narrow spot with a size $L(t)$, amplitude $\aleph(t)$, and a characteristic value $K(t)$ of the field's wavenumber [18]. The conservation of the norm (8) imposes a restriction $\aleph^{2} L \sim N$, i.e., $L \sim N / \aleph^{2}$. Next, the self-focusing part (9) of the Hamiltonian (9), which drives the collapse, can be estimated as

$$
H_{\text {focus }} \sim-\aleph^{4} L \sim-N \aleph^{2} .
$$

On the other hand, the collapse can be checked by the gradient term (10) in the full Hamiltonian, that, in the same approximation, can be estimated as $H_{\text {grad }} \sim \aleph^{2} K L \sim N K$. Further, Eqs. (4) - (6) suggest an estimate $K \sim \aleph^{2}$ for the characteristic wavenumber of the wave field (the same estimate for $K$ follows from an expression (20) for the exact stationary-soliton solution given below), thus we have $H_{\text {grad }} \sim N \aleph^{2}$. Comparing this with the expression (13), one concludes that the parts of the Hamiltonian promoting and inhibiting the collapse scale the same way as $\aleph \rightarrow \infty$ (or $L \rightarrow 0$ ), hence a weak collapse [18] may be possible (but not necessarily) in systems of the present type. In the models of GSs studied thus far and based on GMTM, collapse has never been reported. The real existence of the collapse in the present one-dimensional three-wave GS model is therefore a novel dynamical feature, and it seems quite natural that cuspons and peakons, in the case when they are unstable, play the role of a catalyst stimulating the onset of the collapse.

\section{Analytical solutions}

\subsection{The dispersion relation}

The first step in the investigation of the system is to understand its linear spectrum. Substituting $u_{1,2,3} \sim \exp (i k x-i \omega t)$ into Eqs. (4 -6), and omitting nonlinear terms, we arrive at a dispersion equation,

$$
\left(\omega^{2}-k^{2}-1\right)\left(\omega-\omega_{0}\right)=2 \kappa^{2}(\omega-1) .
$$


If $\kappa=0$, the third wave decouples, and the coupling between the first two waves produces a commonly known gap, so that the solutions to Eq. (14) are $\omega_{1,2}= \pm \sqrt{1+k^{2}}$ and $\omega_{3}=\omega_{0}$. If $\kappa \neq 0$, the spectrum can be easily understood by treating $\kappa$ as a small parameter. However, the following analysis is valid for all values of $\kappa$ in the range $0<\kappa^{2}<1$.

First, consider the situation when $k=0$. Three solutions of Eq. (14) are then

$$
\omega=1, \omega=\omega_{ \pm} \equiv\left(\omega_{0}-1\right) / 2 \pm \sqrt{\left(\omega_{0}+1\right)^{2} / 4+2 \kappa^{2}} .
$$

It can be easily shown that $\omega_{-}<\min \left\{\omega_{0},-1\right\} \leq \max \left\{\omega_{0},-1\right\}<\omega_{+}$, so that one always has $\omega_{-}<-1$, while $\omega_{+} \lessgtr 1$ if $1-\omega_{0} \lessgtr \kappa^{2}$. Next, it is readily seen that, as $k^{2} \rightarrow \infty$, either $\omega^{2} \approx k^{2}$, or $\omega \approx \omega_{0}$. It can also be shown that each branch of the dispersion relation generated by Eq. (14) is a monotonic function of $k^{2}$. Generic examples of the spectrum are shown in Fig. 1, where the panels (a) and (b) pertain, respectively, to the cases $\omega_{0}<1-\kappa^{2}$ with $\omega_{+}<1$, and $\omega_{0}>1$ with $\omega_{+}>1$. The intermediate case, $1-\kappa^{2}<\omega_{0}<1$, is similar to that shown in panel (a), but with the points $\omega_{+}$and 11 at $k=0$ interchanged. When $\omega_{0}<1$, the upper gap in the spectrum is $\min \left\{\omega_{+}, 1\right\}<\omega<\max \left\{\omega_{+}, 1\right\}$, while the lower gap is $\omega_{-}<\omega<\omega_{0}$ When $\omega_{0}>1$, the upper gap is $\omega_{0}<\omega<\omega_{+}$, and the lower one is $\omega_{-}<\omega<1$.

\subsection{Gap solitons}

The next step is to search for GS solutions to the full nonlinear system. In this work, we confine ourselves to the case of zero-velocity GS, substituting into Eqs. (4) - (6)

$$
u_{n}(x, t)=U_{n}(x) \exp (-i \omega t), n=1,2,3,
$$

where it is assumed that the soliton's frequency $\omega$ belongs to one of the gaps. In fact, even the description of zero-velocity solitons is quite complicated. Note, however, that if one sets $\kappa=0$ in Eqs. (4) - (6), keeping nonlinear XPM couplings between the first two and third waves, the gap which exists in the twowave GMT model remains unchanged, and the corresponding family of GS solutions does not essentially alter, in accord with the principle that nonlinear couplings cannot alter gaps or open a new one if the linear coupling is absent [10]; nevertheless, the situation is essentially different if $\kappa$ is vanishingly small, but not exactly equal to zero, see below.

First, we see that the substitution of (16) into Eqs. (4 ) and (5) leads to a system of two ordinary 
differential equations for $U_{1}(x)$ and $U_{2}(x)$,

$$
\begin{gathered}
i U_{1}^{\prime}=\omega U_{1}+U_{2}+\kappa U_{3}+\alpha\left(\alpha \sigma_{1}\left|U_{1}\right|^{2}+\alpha\left|U_{2}\right|^{2}+\left|U_{3}\right|^{2}\right) U_{1} \\
-i U_{2}^{\prime}=\omega U_{2}+U_{1}+\kappa U_{3}+\alpha\left(\alpha \sigma_{1}\left|U_{2}\right|^{2}+\alpha\left|U_{1}\right|^{2}+\left|U_{3}\right|^{2}\right) U_{2},
\end{gathered}
$$

where the prime represents $d / d x$. To solve these equations, we substitute $U_{1,2}=A_{1,2}(x) \exp \left(i \phi_{1,2}(x)\right)$ with real $A_{n}$ and $\phi_{n}$. After simple manipulations, it can be found that $\left(A_{1}^{2}-A_{2}^{2}\right)^{\prime}=0$ and $\left(\phi_{1}+\phi_{2}\right)^{\prime}=0$. With regard to the condition that the soliton fields vanish at infinity, we immediately conclude that

$$
A_{1}^{2}(x)=A_{2}^{2}(x) \equiv S(x)
$$

as for the constant value of $\phi_{1}+\phi_{2}$, it may be set equal to zero without restriction of the generality, so that $\phi_{1}(x)=-\phi_{2}(x) \equiv \phi(x) / 2$, where $\phi(x)$ is the relative phase of the two fields. After this, we obtain two equations for $S$ and $\phi(x)$ from Eqs. (17) and (18),

$$
\begin{gathered}
\phi^{\prime}=-2 \omega-2 \cos \phi-2 \alpha^{2}\left(1+\sigma_{1}\right) S-\frac{U_{3}^{2}}{S}\left(\omega_{0}-\omega-\sigma_{3} U_{3}^{2}\right), \\
S^{\prime}=-2 S \sin \phi-2 \kappa \sqrt{S} U_{3} \sin (\phi / 2),
\end{gathered}
$$

and Eq. (6) for the third wave $U_{3}$ takes the form of a cubic algebraic equation

$$
U_{3}\left(\omega_{0}-\omega-2 \alpha S-\sigma_{3}\left|U_{3}\right|^{2}\right)=2 \kappa \sqrt{S} \cos (\phi / 2)
$$

from which it follows that $U_{3}$ is a real-valued function.

It is relevant to mention here that the above analytical consideration can be readily extended for more general equations (4) and (5) that do not assume the symmetry between the waves $u_{1}$ and $u_{2}$, i.e., the group-velocity terms in the equations are altered as per Eq. (7). In particular, the relation (19) is then replaced by $c_{1} A_{1}^{2}(x)=c_{2} A_{2}^{2}(x) \equiv S(x)$. It can be checked that results for the asymmetric model are not qualitatively different from those presented below for the symmetric one.

Equations (20) and (21) have a Hamiltonian structure, as they can be represented in the form

$$
\frac{d S}{d x}=\frac{\partial H}{\partial \phi}, \quad \frac{d \phi}{d x}=-\frac{\partial H}{\partial S},
$$

with Hamiltonian,

$$
H=2 S \cos \phi+\alpha^{2}\left(1+\sigma_{1}\right) S^{2}+2 \omega S+U_{3}^{2}\left(\omega_{0}-\omega-2 \alpha S\right)-\frac{3}{2} \sigma_{3} U_{3}^{4}
$$


which is precisely a reduction of the Hamiltonian (9) of the original system (4) - (6) for the solutions of the present type. Note that $H$ is here regarded as a function of $S$ and $\phi$, and the relation (22) is regarded as determining $U_{3}$ in terms of $S$ and $\phi$. For soliton solutions, the boundary conditions at $x= \pm \infty$ yield $H=0$, so that the solutions can be obtained in an implicit form,

$$
2 S \cos \phi+\alpha^{2}\left(1+\sigma_{1}\right) S^{2}+2 \omega S+U_{3}^{2}\left(\omega_{0}-\omega-2 \alpha S\right)-(3 / 2) \sigma_{3} U_{3}^{4}=0 .
$$

In principle, one can use the relations (22) and (25) to eliminate $U_{3}$ and $\phi$ and so obtain a single equation for $S$. However, this is not easily done unless $\sigma_{3}=0$ (no SPM term in Eq. (6)), therefore we proceed to examine this special, but important, case first. Note that the no-SPM case also plays an important role for GMTM, which is exactly integrable by means of the inverse scattering transform just in this case [1].

\subsection{Cuspons, the case $\sigma_{3}=0$}

Setting $\sigma_{3}=0$ makes it possible to solve Eq. (22) for $U_{3}$ explicitly in terms of $S$ and $\phi$,

$$
U_{3}=\frac{2 \kappa \sqrt{S} \cos (\phi / 2)}{\omega_{0}-\omega-2 \alpha S} .
$$

For simplicity, we also set $\sigma_{1}=0$ in Eqs. (4) and (5) and subsequent equations, although the latter assumption is not crucially important for the analysis developed below, and if relaxed, the conclusions of this subsection would not be substantially altered.

As the next step, one can also eliminate $\phi$, using Eqs. (25 ) and (26), to derive a single equation for $S$,

$$
\begin{gathered}
(d S / d x)^{2}=4 S^{2} F(S), \\
\text { where } \quad F(S) \equiv\left(1-\omega-\frac{1}{2} \alpha^{2} S\right)\left[2\left(1+\frac{\kappa^{2}}{\omega_{0}-\omega-2 \alpha S}\right)-\left(1-\omega-\frac{1}{2} \alpha^{2} S\right)\right] .
\end{gathered}
$$

The function $F(S)$ has either one or three real zeros $S_{0}$. One is

$$
S_{01}=2(1-\omega) / \alpha^{2}
$$

and the remaining two, if they exist, are real roots of the quadratic equation,

$$
\left(2+2 \omega+\alpha^{2} S_{0}\right)\left(\omega_{0}-\omega-2 \alpha S_{0}\right)+4 \kappa^{2}=0 .
$$

Only the smallest positive real root of Eq. (30), to be denoted $S_{02}$ (if such exists), will be relevant below. Note, incidentally, that $F(S)$ cannot have double roots. It is easy to see that a consequence of this fact is that Eq. (27) cannot generate kink solutions, which have different limits as $x \rightarrow \pm \infty$. 
For a soliton solution of $(27)$, we need first that $F(0)>0$, which can be shown to be exactly equivalent to requiring that $\omega$ belongs to either the upper or the lower gap of the linear spectrum. We note that the coupling to the third wave gives rise to the rational nonlinearity in the expression (28), despite the fact that the underlying system (4) - (6) contains only cubic nonlinear terms. Even if the coupling constant $\kappa$ is small, it is clear that the rational nonlinearity may produce a strong effect in a vicinity of the critical value of the squared amplitude, at which the denominator in the expression (28) vanishes,

$$
S_{\text {cr }}=\left(\omega_{0}-\omega\right) / 2 \alpha,
$$

where one must have $\alpha\left(\omega_{0}-\omega\right)>0$ for $S_{\mathrm{cr}}>0$ (otherwise, the critical value does not exist). At $S=S_{\mathrm{cr}}$, $F(S)$ is singular.

In the case when $S_{\text {cr }}$ exists, the structure of the soliton crucially depends on whether, with an increase of $S$, the function $F(S)$ defined by Eq. (28) first reaches zero at $S=S_{0}$, or, instead, it first reaches the singularity at $S=S_{\mathrm{cr}}$, i.e., whether $0<S_{0}<S_{\mathrm{cr}}$, or $0<S_{\mathrm{cr}}<S_{0}$. In the former case, the existence of $S_{\text {cr }}$ plays no role, and the soliton is a regular one, having the amplitude $\sqrt{S_{0}}$. This soliton may be regarded as obtained by a smooth deformation from the usual GS known in GMTM at $\kappa=0$.

As the soliton cannot have an amplitude larger than $\sqrt{S_{\mathrm{cr}}}$, in the case $0<S_{\mathrm{cr}}<S_{0}$ the amplitude takes this critical value. The soliton is singular in this case, being a cuspon, (see details below), but, nevertheless, it is an absolutely relevant solution. If $S_{\mathrm{cr}}<0$ and $S_{0}>0$ or vice versa, then the soliton may only be, respectively, regular or singular, and no soliton exists if both $S_{0}$ and $S_{\mathrm{cr}}$ are negative. Further, it is readily shown that for all these soliton solutions, $S(x)$ is symmetric about its center, which may be set at $x=0$, that is, $S(x)$ is an even function of $x$. For the cuspon solutions, and for those regular solutions whose squared amplitude is $S_{01}$, it can also be shown that the phase variable $\psi(x)=\phi(x)-\pi$ and $U_{3}(x)$ are odd functions of $x$, while for those regular solutions whose amplitude is $S_{02}$ the phase variable $\phi(x)$ and $U_{3}(x)$ are, respectively, odd and even functions of $x$.

It is now necessary to determine which parameter combinations in the set $\left(\omega, \omega_{0}, \alpha\right)$ permit the options described above. The most interesting case occurs when $\omega_{0}>\omega$ (so that $\omega$ must lie in the lower gap, see Fig. 1) and $\alpha>0$ (the latter condition always holds in the applications to nonlinear optics). In this case, it can be shown that the possible root $S_{02}$ of Eq. (30) is not relevant, and the options are determined by 
the competition between $S_{01}$ and $S_{\mathrm{cr}}$. The soliton is a cuspon $\left(0<S_{\mathrm{cr}}<S_{01}\right)$ if

$$
\alpha\left(\omega_{0}-\omega\right)<4(1-\omega) .
$$

In effect, the condition (32) sets an upper bound on $\alpha$ for given $\omega_{0}$ and $\omega$, which is always satisfied if $0<\alpha<4$.

If, on the other hand, the condition (32) is not satisfied (i.e., $0<S_{01}<S_{\mathrm{cr}}$ ), we obtain a regular soliton. In a less physically relevant case, when again $\omega_{0}>\omega$ but $\alpha<0$, cuspons cannot occur (as this time $S_{\text {cr }}<0$, see Eq. (31), and only regular solitons may exist.

Next we proceed to the case $\omega_{0}<\omega$, so that $\omega$ is located in the upper gap. For $\alpha>0$, we have $S_{\text {cr }}<0$, hence only regular solitons may occur, and indeed it can be shown that there is always at least one positive root $S_{0}$, so a regular soliton exists indeed. If $\alpha<0$, then we have $S_{\text {cr }}>0$, but it can be shown that, if $\omega_{0}<1-\kappa^{2}$ (when also $\omega<1$ ), there is at least one positive root $S_{0}<S_{\text {cr }}$; thus, only a regular soliton can exist in this case too. On the other hand, if $\alpha<0$ and $\omega_{0}>1-\kappa^{2}$ (and then $\omega>1$ ), there are no positive roots $S_{0}$, and so only cuspons occur.

Let us now turn to a detailed description of the cuspon's local structure near its center, when $S$ is close to $S_{\mathrm{cr}}$. From the above analysis, one sees that cuspons occur whenever $\omega$ lies in the lower gap, with $\omega_{0}>\omega$ and $\alpha>0$, so that the criterion (32) is satisfied, or when $\omega$ lies in the upper gap with $1-\kappa^{2}<\omega_{0}<\omega$ and $\alpha<0$. To analyze the structure of the cuspon, we first note that, as it follows from Eq. (25), one has $\cos \phi=-1$ (and so $\phi=\pi$ ) when $S=S_{\text {cr }}$, which suggest to set

$$
S_{\mathrm{cr}}-S \equiv \delta \cdot \kappa^{2} R, \quad 1+\cos \phi \equiv \delta \cdot \rho,
$$

where $\delta$ is a small positive parameter, and the stretched variables $R$ and $\rho$ are positive. It then follows from Eq. (25) that, to the leading order in $\delta, \rho=\rho_{0} R$, where

$$
\rho_{0} \equiv \alpha^{3}\left(S_{01}-S_{\mathrm{cr}}\right),
$$

which must be always positive for a cuspon, as it follows from the above analysis. We also stretch the spatial coordinate, defining $x \equiv \delta^{3 / 2} \kappa^{2} y$, the soliton center being at $x=0$. Note that, since $S(x)$ is an even function of $x$, it is sufficient to set $x>0$ in this analysis. Then, on substituting the first relation from Eq. (33) into Eq. (27), we get, to the leading order in $\delta$, an equation

$$
R(d R / d y)^{2}=\rho_{0} S_{\mathrm{cr}}^{2} / \alpha^{2} \equiv K^{2},
$$




$$
\text { so that } \quad R=(3 K y / 2)^{2 / 3}
$$

Note that in the original unstretched variables, the relation (36) shows that, near the cusp,

$$
\begin{gathered}
S_{\mathrm{cr}}-S(x) \approx(3 K \kappa x / 2)^{2 / 3}, \\
\text { hence } \quad d S / d x \approx(2 / 3)^{1 / 3}(K \kappa)^{2 / 3} \cdot x^{-1 / 3},
\end{gathered}
$$

and it follows from Eq. (26) that $U_{3}$ is unbounded near the cusp,

$$
U_{3} \approx\left(S_{\mathrm{cr}} / \alpha\right)\left(2 \alpha \rho_{0} K^{2} / 3 \kappa x\right)^{1 / 3}
$$

In particular, Eq. (38) implies that, as $K \kappa$ decreases, the cusp gets localized in a narrow region where $|x| \lesssim K^{2} \kappa^{2}$ (outside this region, $|d S / d x|$ is bounded and shows no cusp). Note that this limit can be obtained either as $\kappa^{2} \rightarrow 0$, or as $\rho_{0} \rightarrow 0$ (recall that $\rho_{0}$ is defined in Eq. (34)).

An example of the cuspon is shown in Fig. 2. Although the first derivative in the cuspon is singular at its center, as follows from Eq. (38 ) (see also Fig. 2(a)), it is easily verified that the value of the Hamiltonian (9) (and, obviously, the value of the norm (8) too) is finite for the cuspon solution. These solitons are similar to cuspons found as exact solutions to the Camassa-Holm (CH) equation [13, 14], which have a singularity of the type $|x|^{1 / 3}$ or $|x|^{2 / 3}$ as $|x| \rightarrow 0$, cf. Eqs. (37) and (38). The CH equation is integrable, and it is degenerate in the sense that it has no linear terms except for $\partial u / \partial t$ (which makes the existence of the solution with a cusp singularity possible). Our three-wave system ( 4) - (6) is not degenerate in that sense; nevertheless, the cuspon solitons are possible in it because of the model's multicomponent structure: the elimination of the third component generates the non-polynomial nonlinearity in Eqs. (17), (18), and, finally, in Eqs. (21) and (27), which gives rise to the cusp. It is noteworthy that, as well as the $\mathrm{CH}$ model, ours gives rise to two different coexisting families of solitons, viz., regular ones and cuspons. It will be shown below that the solitons of both types may be stable.

In the special case $\kappa \ll 1$, when the third component is weakly coupled to the first two ones in the linear approximation, a straightforward perturbation analysis shows that our cuspons look like peakons; that is, except for the above-mentioned narrow region of the width $|x| \sim \kappa^{2}$, where the cusp is located, they have the shape of a soliton with a discontinuity in the first derivative of $S(x)$ and a jump in the phase $\phi(x)$, which are the defining features of peakons $([13,15])$. An important result of our analysis is that the family of solitons obtained in the limit $\kappa \rightarrow 0$ is drastically different from that in the model 
where one sets $\kappa=0$ from the very beginning. In particular, in the most relevant case, with $\omega_{0}>\omega$ and $\alpha>0$, the family corresponding to $\kappa \rightarrow 0$ contains regular solitons whose amplitude is smaller than $\sqrt{S_{\mathrm{cr}}}$; however, the solitons whose amplitude at $\kappa=0$ is larger than $\sqrt{S_{\mathrm{cr}}}$, i.e., the ones whose frequencies belong to the range (32) (note that the definition of $S_{\text {cr }}$ does not depend on $\kappa$ at all, see Eq. (31)), are replaced by the peakons which are constructed in a very simple way; drop the part of the usual soliton above the critical level $S=S_{\text {cr }}$, and bring together the two symmetric parts which remain below the critical level, see Fig. 2(b). It is interesting that peakons are known as exact solutions to a version of the integrable $\mathrm{CH}$ equation slightly different from that which gives rise to the cuspons. As well as in the present system, in that equation the peakons coexist with regular solitons [15]. In the next subsection, we demonstrate that peakons, which are found here only as limit-form solutions in the no-SPM case, $\sigma_{3}=0$, become generic solutions in the case $\sigma_{3} \neq 0$.

\subsection{Peakons, the case $\sigma_{3} \neq 0$}

A natural question is whether the cuspon solutions are structurally stable. Here, we address this issue by restoring the SPM term in Eq. (6), that is, we now set $\sigma_{3} \neq 0$, but assume that it is a small parameter. Note that, in the application to nonlinear optics. we would expect that $\sigma_{3}>0$, but in any application to the flow of a density-stratified fluid, there is no such a restriction on the sign of $\sigma_{3}$. We still keep $\sigma_{1}=0$, as the inclusion of the corresponding SPM terms in Eqs. (4) and (5) amounts to trivial changes both in the above analysis, and in that presented below. On the other hand, we show below that the inclusion of the SPM term in Eq. (6 ) is a structural perturbation which drastically changes the character of the soliton solutions.

In view of the above results concerning the cuspons, we shall restrict our discussion here to the most interesting case when $S(x)$ is an even function of $x$, while $\psi(x)=\phi(x)-\pi$ and $U_{3}(x)$ are odd functions. In principle, one can use the relations (22) and (25) to eliminate $\phi$ and $U_{3}$ and so obtain a single equation for $S$ (a counterpart to Eq. (27)), as was done above when $\sigma_{3}=0$. However, when $\sigma_{3} \neq 0$ it is not possible to do this explicitly. Hence, instead we shall develop an asymptotic analysis valid for $x \rightarrow 0$, together with numerical results obtained by direct numerical integration of Eqs. (20) and (21), subject of course to the constraints (22) and (25). Since singularities only arise at the center of the soliton (i.e., at $x=0$ ) when $\sigma_{3}=0$, it is clear that the introduction of a small $\sigma_{3} \neq 0$ will produce only a small 
deformation of the soliton solution in the region where $x$ is bounded away from zero.

First, we consider regular solitons. Because the left-hand side of Eq. (22) is not singular at any $x$, including the point $x=0$ when $\sigma_{3}=0$, we expect that regular solitons survive a perturbation induced by $\sigma_{3} \neq 0$. Indeed, if there exists a regular soliton, with $S_{0} \equiv S(x=0)$, and $\phi(x=0)=\pi$ and $U_{3}(x=0)=0$, it follows from Eq. (25) that the soliton's amplitude remains exactly the same as it was for $\sigma_{3}=0$, due to the fact that the regular soliton has $U_{3}(x=0)=0$.

Next, we turn to the possibility of singular solutions, that is, cuspons or peakons. Since we are assuming that $S_{0}=S(x=0)$ is finite, and that $\phi(x=0)=\pi$, it immediately follows from Eq. (22) that when $\sigma_{3} \neq 0, U_{3}$ remains finite for all $x$, taking some value $U_{0}$ say as $x \rightarrow 0+$. Note that since $U_{3}$ is an odd function of $x$, in the case of interest when $U_{0} \neq 0$, there is a discontinuity in $U_{3}$ at $x=0$, i.e., a jump from $U_{0}$ to $-U_{0}$. This is in marked contrast to the cuspons for which $U_{3}$ is infinite at the center, see Eq. (39). Further, it then follows from (21) that, as $x \rightarrow 0$, there is a discontinuity in $(d S / d x)$ with a jump from $2 \kappa U_{0} \sqrt{S_{0}}$ to $-2 \kappa U_{0} \sqrt{S_{0}}$. Hence, if we can find soliton solutions of this type, with $U_{0} \neq 0$, they are necessarily peakons, and we infer that cuspons do not survive the structural perturbation induced by $\sigma_{3} \neq 0$. Indeed, if we assume that $U_{0} \neq 0$ then Eq. (22), taken in the limit $x \rightarrow 0$, immediately shows that

$$
2 \alpha\left(S_{\mathrm{cr}}-S_{0}\right)=\sigma_{3} U_{0}^{2}
$$

(recall that $S_{\text {cr }}$ is defined by Eq. (31)). Further, the Hamiltonian relation (25), also taken in the limit $x \rightarrow 0$, shows that

$$
-\frac{\rho_{0}}{\alpha} S_{0}-\alpha^{2} S_{0}\left(S_{\mathrm{cr}}-S_{0}\right)=\frac{1}{2} \sigma_{3} U_{0}^{4},
$$

where we have used Eq. (40) (recall that $\rho_{0}$ is defined by Eq. (34)). Elimination of $U_{0}$ from $(40,41)$ yields a quadratic equation for $S_{0}$, whose positive roots represent the possible crest values for a peakon.

We recall that for a cuspon which exists when $\sigma_{3}=0$, one has $\rho_{0}>0$, that is, the the amplitude of the corresponding formal regular soliton exceeds the critical value of the amplitude, see Eq. (34). Then, on retaining this condition that $\rho_{0}>0$, it immediately follows from Eqs. (40) and (41) that no peakons may exist if the SPM coefficient in Eq. (6) is positive, that is, $\sigma_{3}>0$. Indeed, Eq. (40) shows that $S_{\mathrm{cr}}-S_{0}>0$ if $\sigma_{3}>0$, which, along with $\rho_{0}>0$, leads to a contradiction in the relation (41).

Further, it is easy to see that a general necessary condition for the existence of peakons follows from 
Eqs. (40) and (41), namely

$$
\sigma_{3} \rho_{0}<0
$$

Hence peakons are possible if $\sigma_{3}<0$, or if we keep $\sigma_{3}>0$ but allow $\rho_{0}<0$. In the remainder of this section, we will show that peakons may exist only if $\rho_{0}>0$. Hence, it follows from the necessary condition (42), that peakons may indeed be possible solely in the case $\sigma_{3}<0$. On the other hand, regular solitons do exist in the case $\sigma_{3}>0$ (i.e., in particular, in nonlinear-optics models), as they have $U_{0}=0$, and so neither Eq. (40) nor its consequence in the form of the inequality (42) apply to regular solitons. The existence of peakons for $\sigma_{3}<0$, and of regular solitons for $\sigma_{3}>0$ will be confirmed by direct numerical results presented in the next section.

To obtain a necessary condition (which will take the form of $\rho_{0}>0$ ) for the existence of peakons, we notice that existence of any solitary wave implies the presence of closed dynamical trajectories in the phase plane of the corresponding dynamical system, which is here based on the ordinary differential equations (20) and (21), supplemented by the constraint (22). Further, at least one stable fixed point (FP) must exist inside these closed trajectories, and therefore the existence of such a stable FP is a necessary condition for the existence of any solitary wave.

The FP are found by equating to zero the right-hand sides of Eq. (20) and (21), which together with the constraint then (22) give three equations for three co-ordinates $\phi, S$ and $U_{3}$ of the FP. First of all, one can find a trivial unstable FP of the dynamical system,

$$
\cos \phi=-\frac{\omega+\kappa^{2} /\left(\omega_{0}-\omega\right)}{1+\kappa^{2} /\left(\omega_{0}-\omega\right)}, \quad S=0,
$$

which does not depend on $\sigma_{3}$, and is of course the fixed point obtained by the solitary wave as $x \rightarrow \pm \infty$. The coordinates $\phi_{*}, S_{*}$ and $U_{3 *}$ of the non-trivial FP are then given by,

$$
\begin{gathered}
\phi_{*}^{(1)}=\pi, \quad S_{*}^{(1)}=\frac{1-\omega}{\alpha^{2}}=\frac{1}{2} S_{01}, \quad U_{3 *}^{(1)}=0, \\
\phi_{*}^{(2)}=\pi, \quad\left(2-\sigma_{3}\right) S_{*}^{(2)}=2 S_{\mathrm{cr}}-\frac{\sigma_{3}}{2} S_{01}, \quad\left(2-\sigma_{3}\right)\left(\alpha U_{3 *}^{(2)}\right)^{2}=\rho_{0}-\alpha^{3} S_{\mathrm{cr}}, \\
\text { and }\left(2-\sigma_{3}\right) S_{*}^{(3)}=2 S_{\mathrm{cr}}-\frac{\sigma_{3}}{2} S_{01}+\frac{\kappa^{2}}{\alpha}, \quad\left(2-\sigma_{3}\right)\left(\alpha U_{3 *}^{(3)}\right)^{2}=\rho_{0}-\alpha^{3} S_{\mathrm{cr}}-\alpha^{2} \kappa^{2}, \\
\kappa U_{3 *}^{(3)}=-2 \sqrt{S_{*}^{(3)}} \cos \phi_{*}^{(3)} / 2 .
\end{gathered}
$$

To be specific we now consider the case of most interest, when both $S_{01}>0$ and $S_{\mathrm{cr}}>0$. In this case the $\mathrm{FP}^{(1)}$ given by $(43)$ exists for all $\sigma_{3}$ and all $\rho_{0}$. However, for small $\sigma_{3}$ (in fact $\sigma_{3}<2$ is enough) 
and small $\kappa$, the $\mathrm{FP}^{(2,3)}$ given by (44) and (45) exist only when $\rho_{0}>0$. Indeed, they exist only for $\rho_{0}>\alpha^{3} S_{01}$ and $\rho_{0}>\alpha^{3} S_{01}+\kappa^{2}$ respectively, or, on using the definition (34) of $\rho_{0}$, when $S_{01}>2 S_{\mathrm{cr}}$ and $S_{01}>2 S_{\mathrm{cr}}+\kappa^{2} / \alpha$ respectively.

Let us first suppose that $\rho_{0}<0$. Then there is only the single non-trivial FP, namely $\mathrm{FP}^{(1)}$ given by (43). This is clearly ascociated with the regular solitons, whose amplitude at the crest is $S_{01}$. Hence, we infer that for $\rho_{0}<0$ there are no other solitary wave solutions, and in particular, no peakon solutions (and indeed, no cuspon solutions when $\sigma_{3}=0$ as we found in subsection 2.3 above). When combined with the necessary condition (42) for peakons, we infer that there can be no peakons when $\sigma_{3}>0$, thus excluding peakons from applications to nonlinear optics, where this SPM coefficient is required to be positive. However, peakons may occur in density-stratified fluid flows where there is no inherent restriction on the sign of $\sigma_{3}$, and this case is considered below. But first we note that if we do have, as here, $\rho_{0}<0$ and $\sigma_{3}>0$, then the argument just presented does suggest that there may be periodic solutions with a peakon-type discontinuity at the crest, and indeed our numerical solutions of the system $(20,21)$ suggest that this is the case.

Next we suppose that $\rho_{0}>0$. First, if $S_{01}<2 S_{\text {cr }}$ then there is again just the single non-trivial $\mathrm{FP}$, namely $\mathrm{FP}^{(1)}$ given by (43). But now, by analogy with the existence of cuspons when $\rho_{0}>0$ and $\sigma_{3}=0$ we infer that the solitary wave solution which is associated with this fixed point is a peakon, whose crest amplitude $S_{0}$ for small $\sigma_{3}$ is close to $S_{\mathrm{cr}}$, where we note that $S_{*}^{(1)}=S_{01} / 2<S_{\mathrm{cr}}$. Then, if on the other hand, $S_{01}>2 S_{\text {cr }}$ the $\mathrm{FP}^{(2,3)}$ become available as well. We now infer that the peakon solitary wave solution continues to exist, and for sufficiently small $\sigma_{3}$ and $\kappa$ is associated with $\mathrm{FP}^{(2)}$. Although equation (44) implies $S_{*}^{(2)} \approx S_{\text {cr }}$ and the crest value $S_{0}$ of the peakon, determined by $(40,41)$, is also approximately given by $S_{\mathrm{cr}}$, we nevertheless have $S_{0}>S_{*}^{(2)}$ as required. Note that now the fixed points $\mathrm{F}^{(1)}$ and $\mathrm{F}^{(3)}$ lie outside the peakon solitary wave orbit. We show in Fig. 3 a plot of a typical peakon obtained by numerical solution Eqs. (20) and (21). 


\section{$3 \quad$ Numerical results}

\subsection{Simulation techniques}

The objectives of direct numerical simulations of the underlying equations (4) - (6) were to check the dynamical stability of regular solitons, cuspons, and peakons in the case $\sigma_{3}=0$, and the existence and stability of peakons in the more general case, $\sigma_{3} \neq 0$. Both finite-difference and pseudo-spectral numerical methods have been used, in order to check that the same results are obtained by methods of both types. We used semi-implicit Crank-Nicholson schemes, in which the nonlinear terms were treated by means of the Adams-Bashforth algorithm.

The presence of singularities required a careful treatment of cuspon and peakon solutions. To avoid numerical instabilities due to discontinuities, we sometimes introduced an artificial weak high-wave number viscosity into the pseudospectral code. We have found that viscosities $\mathcal{O}\left(10^{-5}\right)$ were sufficient to avoid the Gibbs' phenomenon in long-time simulations. When instabilities occur at a singular point (cusp or peak), it is hard to determine whether the instability is a real one or a numerical artifact. Therefore we checked the results by means of a finite-difference code which used an adaptive staggered grid; motivated by the analysis of the vicinity of the point $x=0$ reported above, we introduced the variable $\xi \equiv x^{2 / 3}$ to define an adaptive grid, and also redefined $U_{3} \equiv \sqrt{\xi} \widetilde{U}_{3}$. In the rescaled variables, the cusp singularity vanishes. We followed this approach solely to check the possible occurrence of numerical instabilities,

In the following subsections we present some typical examples of our numerical results for both cases considered above, viz., $\sigma_{3}=0$ when cuspons may occur, and $\sigma_{3}<0$ when peakons may occur.

\subsection{The case $\sigma_{3}=0$}

First, we report numerical results obtained for the stability of regular solitary waves in the case $\sigma_{3}=0$. As initial configurations, we used the corresponding stationary solutions to Eqs. (20) and (21). To test the stability of the regular solitary waves, we added small perturbations to them. As could be anticipated, the regular solitary wave emits a small dispersive wave train and relaxes to a stationary soliton, see Fig. 4. If, however, regular solitons are taken at parameter values close to (but within) the parameter boundary of the cuspon region, an initial perturbation does not make the soliton unstable, but it excites persistent internal vibrations in it, see an example in Fig. 5. These and many other simulations clearly show that 
the regular soliton is always stable, and, close to the parameter border with cuspons, it has a persistent internal mode.

As it was shown above in an analytical form, Eqs. (17) and (18) (with $\sigma_{3}=0$ ) support peakons when $\rho_{0}>0$ and $\kappa^{2} \rho_{0}$ is very small. Direct simulations show that the peakons do exist in this case, and may be either unstable or stable. In the case when they are unstable, a high-wavenumber instability develops around the central peak. In Fig. 6, we display the time evolution of a typical stable peakon, while detailed investigation of the case when the peakon is unstabe is left for further work. Next, we consider the case when the initial state is a regular soliton, defined by system parameters which are close to the parameter boundary for peakons but with $\rho_{0}<0$, and allow it to evolve in the system for parameter values which only support peakons and exclude regular solitons (i.e. with $\rho_{0}>0$. Unlike the case shown in Fig. 5, the time evolution now does not exhibit internal vibrations. Instead, the solution slowly decays into radiating waves. This outcome can be explained by the fact that the peakon's norm [see Eq.(8)] turns out to be larger than that of the initial condition in this case, and hence rearrangement into a stable peakon is not possible.

Quite a nontrivial result revealed by the simulations is that cuspons may also be stable, see an example in Fig. 7. We note that there is a small shock wave which is emitted by the cuspon's crest. It seems plausible that this shock wave is generated by some initial perturbation which could be a result of the finite-mesh size in the finite-difference numerical scheme employed for the simulations. In fact, the emission of a small-amplitude shock wave is quite a typical way of the relaxation of both cuspons and peakons to a final stable state.

To further test the stability of the cuspons and peakons, in many cases we allowed the initially generated shock wave to re-enter the integration domain (due to periodic boundary conditions used in the simulations) and interact again with the cuspon or peakon. As a result, the existence of stable solitons of these types has been additionally confirmed, see, for instance, the spatial profile of the soliton obtained after a long evolution (Fig. 8). Both the stability of the cuspon, and the presence of a tiny shock wave are evident in the figure.

However, unlike the regular solitons, which were always found to be stable, cuspons are sometimes unstable. Typically, their instability leads to the spatiotemporal collapse, i.e., formation of a singularity in a finite time (see a discussion of the feasible collapse in systems of the present type, given in the 
Introduction). Simulations of the collapse were possible with the use of an adaptive grid. A typical example of the collapse is shown in Fig. 9. In some other cases, which are not displayed here, the instability of peakons could be quite weak, giving rise to their rearrangement into regular solitons by the shedding of a small amount of radiation.

\subsection{The case $\sigma_{3} \neq 0$}

The predictions of the analysis developed above for the most general case, when the SPM terms are present in the model $\left(\sigma_{3} \neq 0\right)$, were also checked against direct simulations. As a result, we have found, in accord with the predictions, that only regular solitons exist in the case $\sigma_{3}>0$, while in the case $\sigma_{3}<0$ both regular solitons and peakons have been found as generic solutions. Further simulations, details of which are not shown here, demonstrate that both regular solitons and peakons are stable in this present case.

\section{Conclusion}

In this work, we have introduced a generic model of three linearly and nonlinearly coupled waves, which describes a situation when three dispersion curves are close to an intersection at one point. The model was cast into the form of a system of two waves with opposite group velocities that, by itself, gives rise to the usual gap solitons, which is further coupled to a third wave with the zero group velocity (in the laboratory reference frame). Situations of this type are possible in various models of nonlinear optics and density-stratified fluid flow. The consideration was focussed on zero-velocity solitons. In a special case when the self-phase modulation (SPM) is absent in the equation for the third wave, soliton solutions were found in an exact form. It was shown that there are two coexisting generic families of solitons: regular solitons and cuspons. In the special case when the coefficient of the linear coupling between the first two waves and the third one vanishes, cuspons are replaced by peakons. Direct simulations have demonstrated that all the regular soliton are stable (in the case when the regular soliton is close to the border of the cuspon region, it has a persistent internal mode), while cuspons and peakons may be both stable and unstable. If they are unstable, they either shed off some radiation and rearrange themselves into regular solitons, or, in most typical cases, the development of the cuspon's instability initiates onset 
of spatiotemporal collapse. Interestingly, the present system gives the first explicit example of the collapse in one-dimensional gap-soliton models.

The most general version of the model, which includes the self-phase modulation term in the equation for the third wave, has also been considered. Analysis shows that cuspons cannot exist in this case, i.e., cuspons, although being dynamically stable, are structurally unstable. However, depending on the signs of the SPM coefficient and some combination of the system's parameters, it was shown that a generic family of peakon solutions may exist instead. In accord with this prediction, the peakons have been found in direct simulations. The peakons, as well as the regular solitons, are stable in the system including the SPM term.

The next step should be consideration of moving solitons, which is suggested by the well-known fact that the usual two-wave model gives rise to moving gap solitons too [1]. However, in contrast to the two-wave system, one may expect a drastic difference between the zero-velocity and moving solitons in the present three-wave model. This is due to the reappearance of a derivative term in Eq. (6), when it is written for a moving soliton, and so solitons which assume a singularity or jump in the $U_{3}$ component, i.e., both cuspons and peakons, cannot then exist. Nevertheless, one may expect that slowly moving solitons will have approximately the same form as the cuspons and peakons, with the singularity at the central point replaced by a narrow transient layer with a large gradient of the $U_{3}$ field. However, detailed analysis of the moving solitons is beyond the scope of this work.

\section{Acknowledgements}

We would like to thank Tom Bridges, Gianne Derks and Sebastian Reich for valuable discussions. B.A.M. and G.A.G. appreciate hospitality of the University of Loughborough (UK). The work of G.A.G. is supported by a European Commission Grant, contract number HPRN-CT-2000-00113, for the Research Training Network Mechanics and Symmetry in Europe (MASIE) 


\section{References}

[1] C.M. de Sterke and J.E. Sipe, in Progress in Optics, edited by E. Wolf (Elsevier, North-Holland, 1994), Vol. XXXIII, Chap. 3, pp. 205.

[2] R. Grimshaw, J. He, and B.A. Malomed, Physica D 113, 26 (1998).

[3] D.N. Christodoulides and R.I. Joseph, Phys. Rev. Lett. 2 , 1746 (1989); A. Aceves and S. Wabnitz, Phys. Lett. A 141, 37 (1989).

[4] N.G.R. Broderick, D.J. Richardson, R.I. Laming, and M. Ibsen, Opt. Lett. 23, 328 (1998).

[5] B.J. Eggleton et al., Phys. Rev. Lett. 76, 1627 (1996); C.M. de Sterke, B.J. Eggleton, and P.A. Krug, J. Lightwave Technol. 15, 1494 (1997).

[6] R. Grimshaw and B.A. Malomed, Phys. Rev. Lett. 72, 949 (1994).

[7] J. Gear and R. Grimshaw, R. Stud. Appl. Math., 70, 235 (1984).

[8] T. Opatrný, B.A. Malomed, and G. Kurizki, Phys. Rev. E 60, 6137 (1999).

[9] W.C.K. Mak, B.A. Malomed, and P.L. Chu, Phys. Rev. E 586708 (1998).

[10] R. Grimshaw and B.A. Malomed, Phys. Lett. A 198, 205 (1995).

[11] H. Aratyn, J. Phys. A 14, 1313 (1981).

[12] T. Peschel et al., Phys. Rev. E 55, 4730 (1997); J. Yang, B.A. Malomed, and D.J. Kaup, Phys. Rev. Lett. 83, 1958 (1999).

[13] R. Camassa and D.D. Holm, Phys. Rev. Lett. 71, 1661 (1993).

[14] R.A. Kraenkel and A. Zenchuk, J. Phys. A 32, 4733 (1999); M.C. Ferreira, R.A. Kraenkel, and A.I. Zenchuk, J. Phys. A 32, 8665 (1999).

[15] R. Beals and D.H. Sattinger, Inverse Problems 15, L1 (1999).

[16] T.Qian and M.Tang, Chaos, Solitons and Fractals 12, 1347 (2001).

[17] I.V. Barashenkov, D.E. Pelinovsky, and E.V. Zemlyanaya, Phys. Rev. Lett. 80, 5117 (1998); A. De Rossi, C. Conti, and S. Trillo, ibid. 81, 85 (1998). 
[18] L. Bergé, Phys. Rep. 303, 259 (1998). 
Fig. 1. Dispersion curves produced by Eq. (14) in the case $\kappa=0.5$ : (a) $\omega_{0}<1-\kappa^{2}$; (b) $\omega_{0}>1$. The dashed line in each panel is $\omega=\omega_{0}$. The case with $1-\kappa^{2}<\omega_{0}<1$ is similar to the case (a) but with the points $\omega_{+}$and 1 at $k=0$ interchanged.

Fig. 2. The shape of the cuspon for $\alpha=2.0, \omega_{0}=0.1, \omega=-0.5$, and (a) $\kappa=0.5$, i.e., in the general case, and (b) $\kappa=0.1$, i.e., for small $\kappa$. In the case (b) we also show the usual gap soliton (by the dashed line), for which the part above the critical value $S=S_{\mathrm{cr}}$ (shown by the dotted line) should be removed and the remaining parts brought together to form the peakon corresponding to $\rho_{0} \kappa^{2} \rightarrow 0$.

sig4.gnu.000.ps Fig. 3. The shape of the peakon in for the case when $\sigma_{3}<0$. The parameters are $\sigma_{3}=-0.01, \kappa=0.1, \alpha=2.0, \omega_{0}=0.1$, and $\omega=-0.5$. In this case, $\rho_{0}=4.8$.

Fig. 4. The shape of an initially-perturbed regular soliton in the case $\sigma_{3}=0$ at $t=5$, which illustrates the stability of the soliton, and a small radiated field. The plot displayed is the field $\operatorname{Re} U_{1}(x)$. The parameters are $\kappa=0.01, \alpha=1.0, \omega_{0}=0.2$, and $\omega=0.9$.

Fig. 5. Internal vibrations of an initially-perturbed regular soliton, which was taken close to the parameter boundary of the cuspon region. The plot shown is the squared amplitude $a \equiv\left|U_{1}(x=0)\right|^{2}$ of the $U_{1}(x)$ field vs. time. The parameters are $\kappa=0.01, \alpha=1.9, \omega_{0}=1.5$, and $\omega=0.5$, with $\rho_{0}=0.095$ (see Eq. (34)).

Fig. 6. An example of a stable peakon. The plot shown is the field $\operatorname{Im} U_{1}$ vs. $x$ and $t$. The parameters are $\kappa=1.0, \alpha=1.95, \omega_{0}=1.5$, and $\omega=0.5$, with $\rho_{0}=0.04875$.

Fig. 7. An example of a stable cuspon. The plot shown is the field $I m U_{1}$ vs. $x$ and $t$. The parameters are $\kappa=1.0, \alpha=1.0, \omega_{0}=1.5$, and $\omega=0.5$, with $\rho_{0}=0.5$.

Fig. 8. The spatial profile of the stable cuspon at $t=20$. The parameters are the same as in Fig. 7 .

Fig. 9. The spatial profile is shown for an unstable cuspon in terms of $\operatorname{Im} U_{1}$ at $t=10^{-3}$. The transition to collapse is clearly seen. The parameters are $\kappa=0.01, \alpha=1.1, \omega_{0}=0.1$, and $\omega=-0.3$, with $\rho_{0}=2.618$ 
(a)

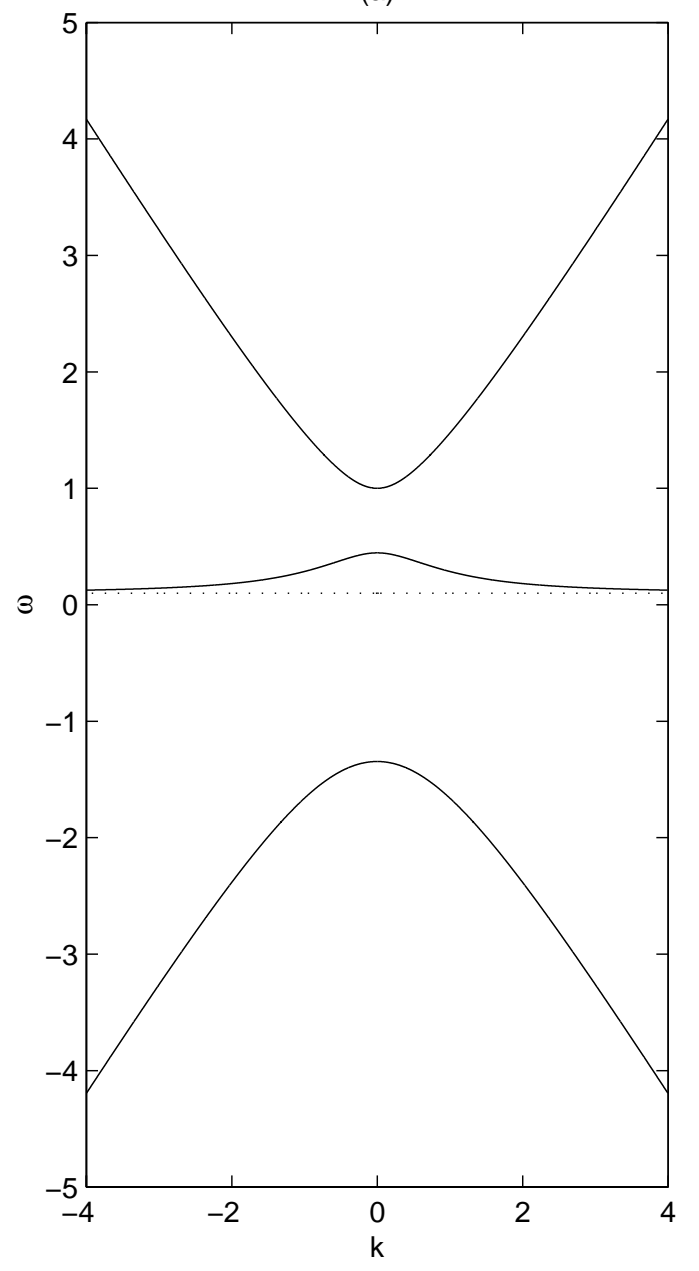

(b)

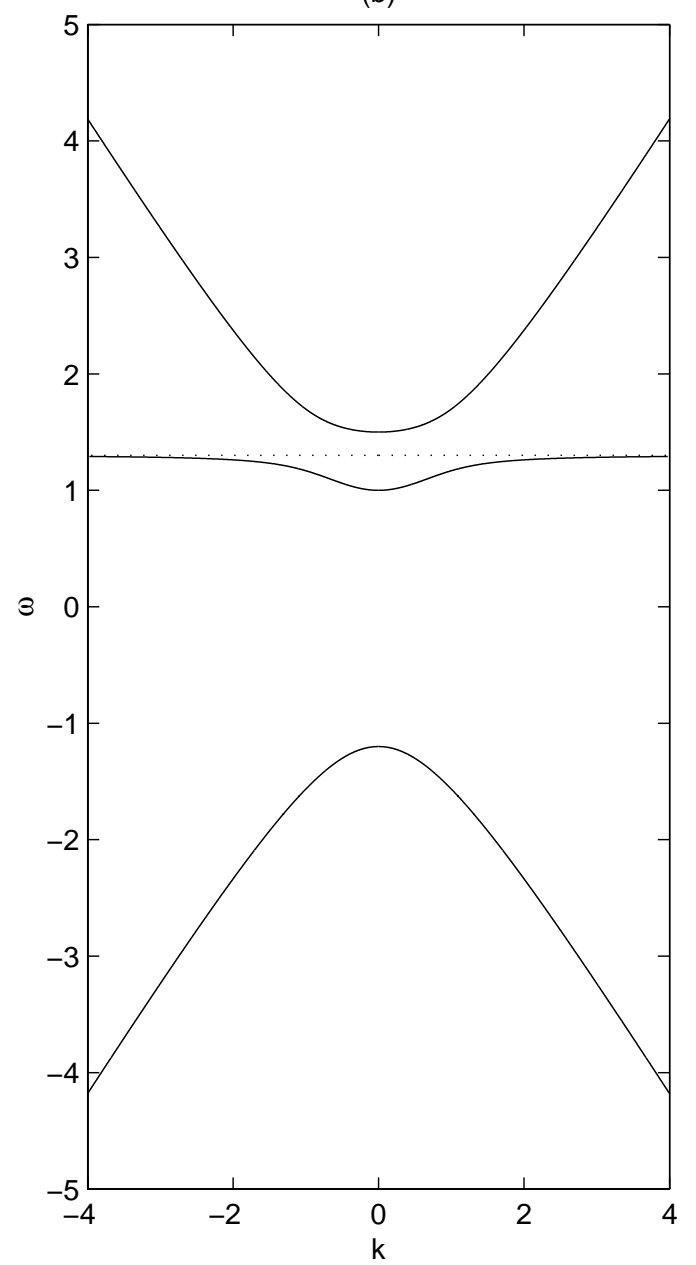

Figure 1: Dispersion curves produced by Eq. (13) in the case $\omega_{0}=0.1$ and $\kappa=0.5$ : (a) $\omega_{0}<1-\kappa^{2}$; (b) $\omega_{0}>1$. The dashed line in each panel is $\omega=\omega_{0}$. The case with $1-\kappa^{2}<\omega_{0}<1$ is similar to case (a) but with the points $\omega_{+}$and 1 at $k=0$ interchanged. 
(a)

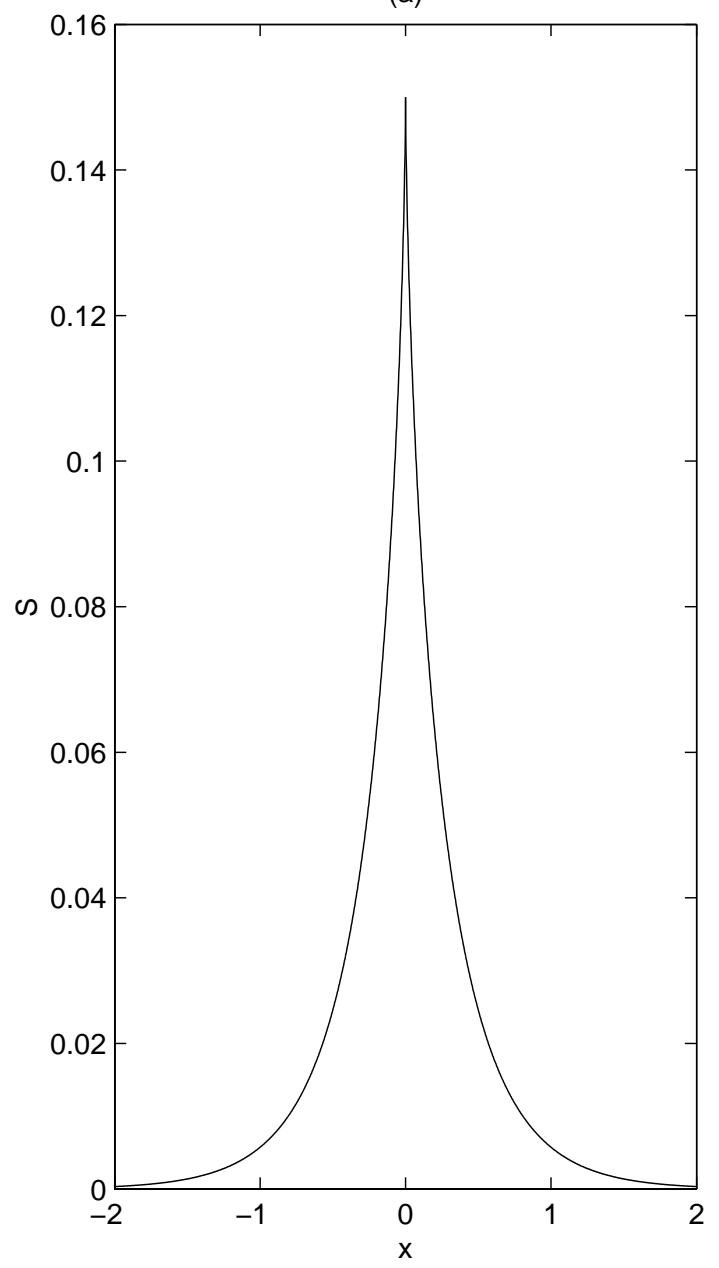

(b)

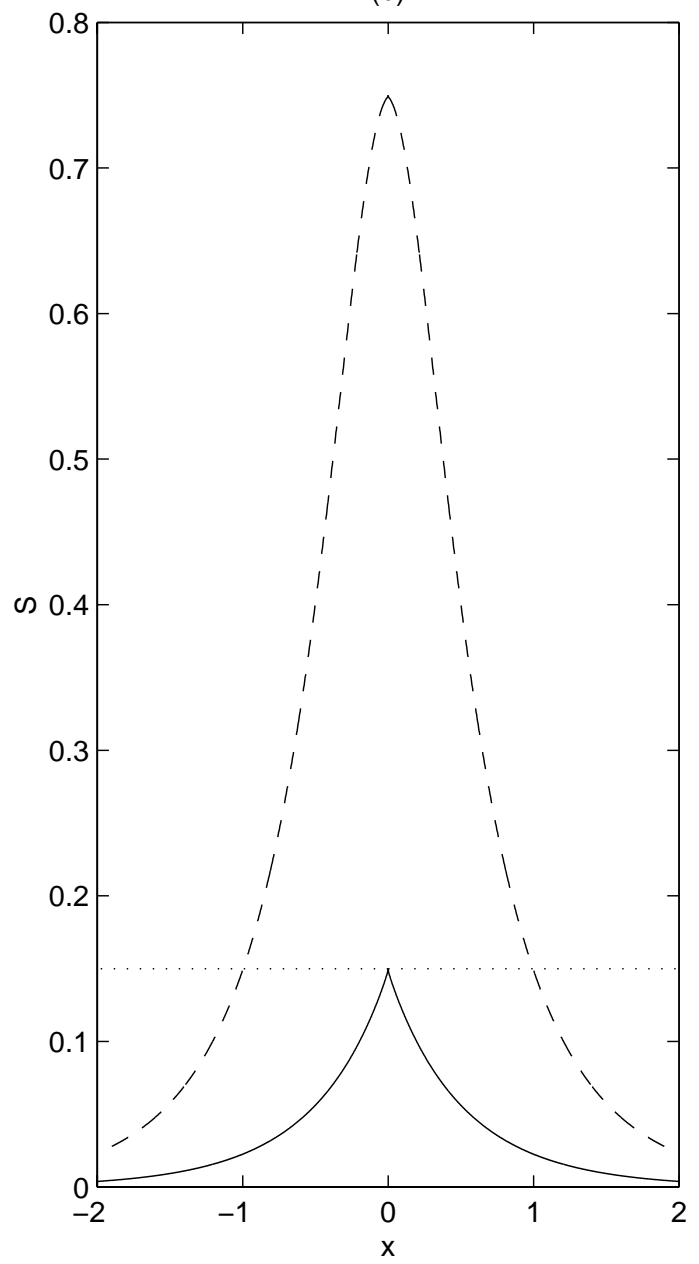

Figure 2: The shape of the cuspon for $\alpha=2.0, \omega_{0}=0.1, \omega=-0.5$, and (a) $\kappa=0.5$, i.e., in the general case, and (b) $\kappa=0.1$, i.e., for small $\kappa$. In the case (b) we also show the usual gap soliton (by the dashed line), for which the part above the critical value $S=S_{\text {cr }}$ (shown by the dotted line) should be removed and the remaining parts brought together to form the peakon corresponding to $\rho_{0} \kappa^{2} \rightarrow 0$. 


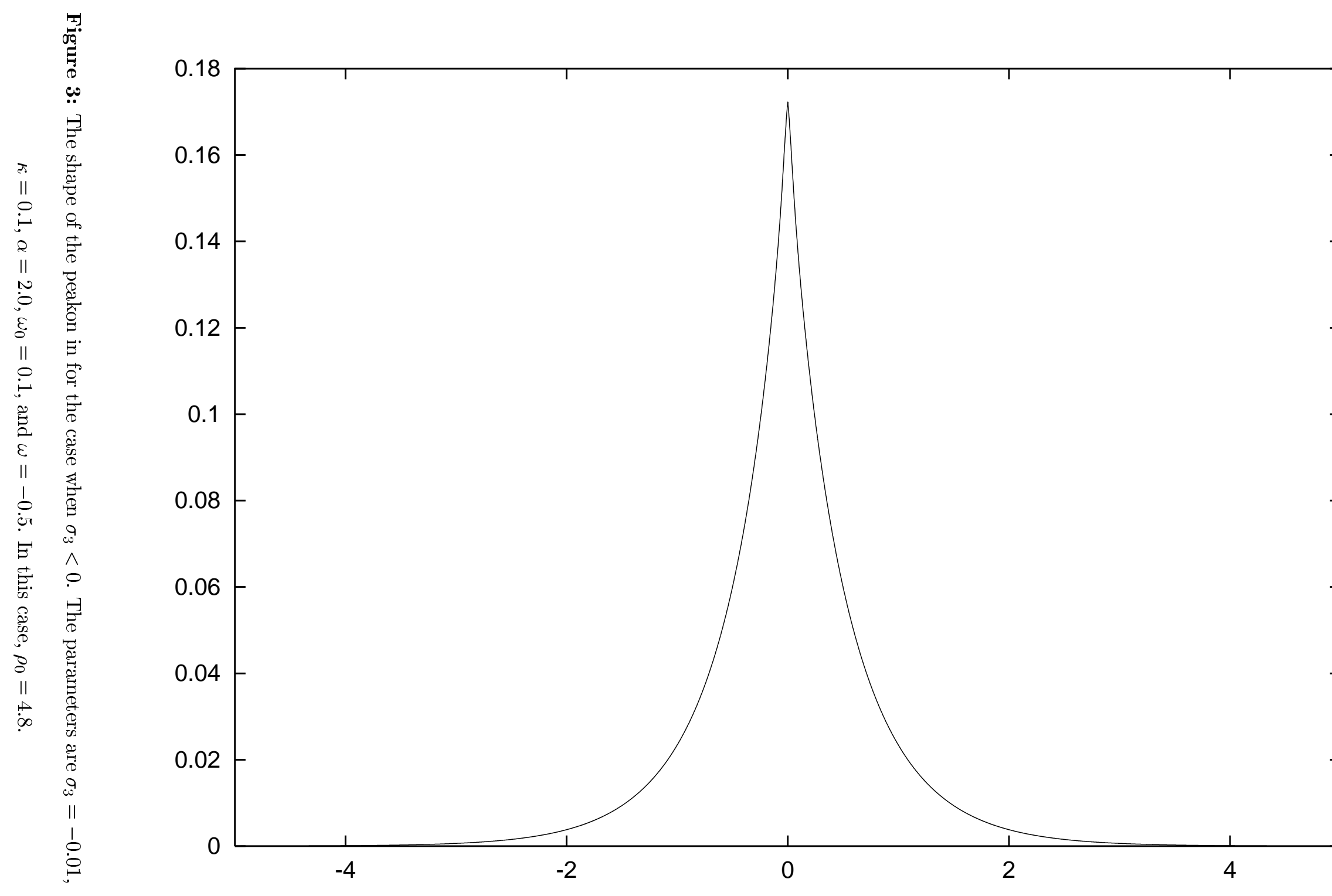




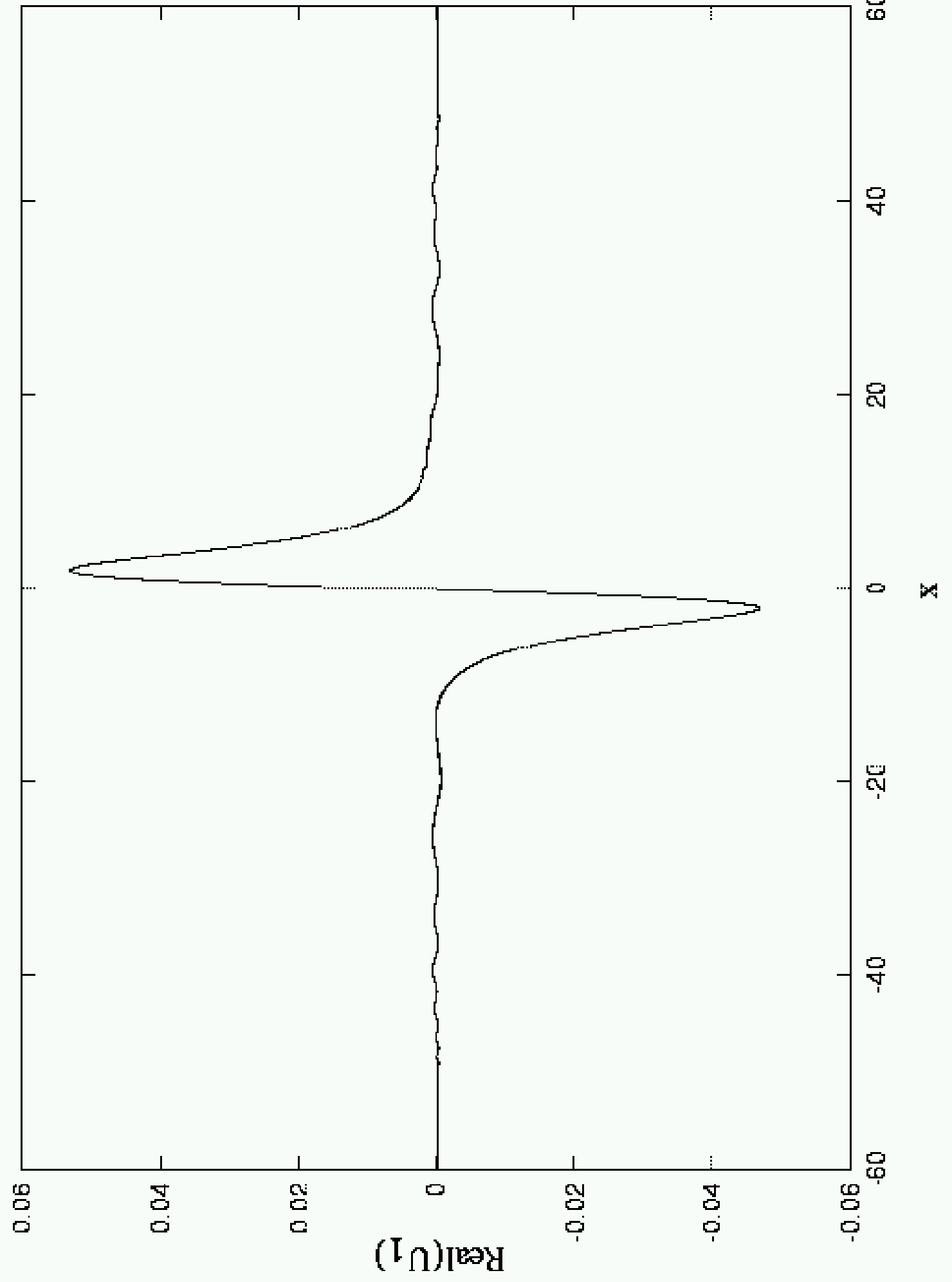

Figure 4: The shape of an initially-perturbed regular soliton in the case $\sigma_{3}=0$ at $t=5$, which illustrates the stability of the soliton, and a small radiated field. The plot displayed is the field $\operatorname{Re} U_{1}(x)$. The parameters are $\kappa=0.01, \alpha=1.0, \omega_{0}=0.2$, and $\omega=0.9$. 


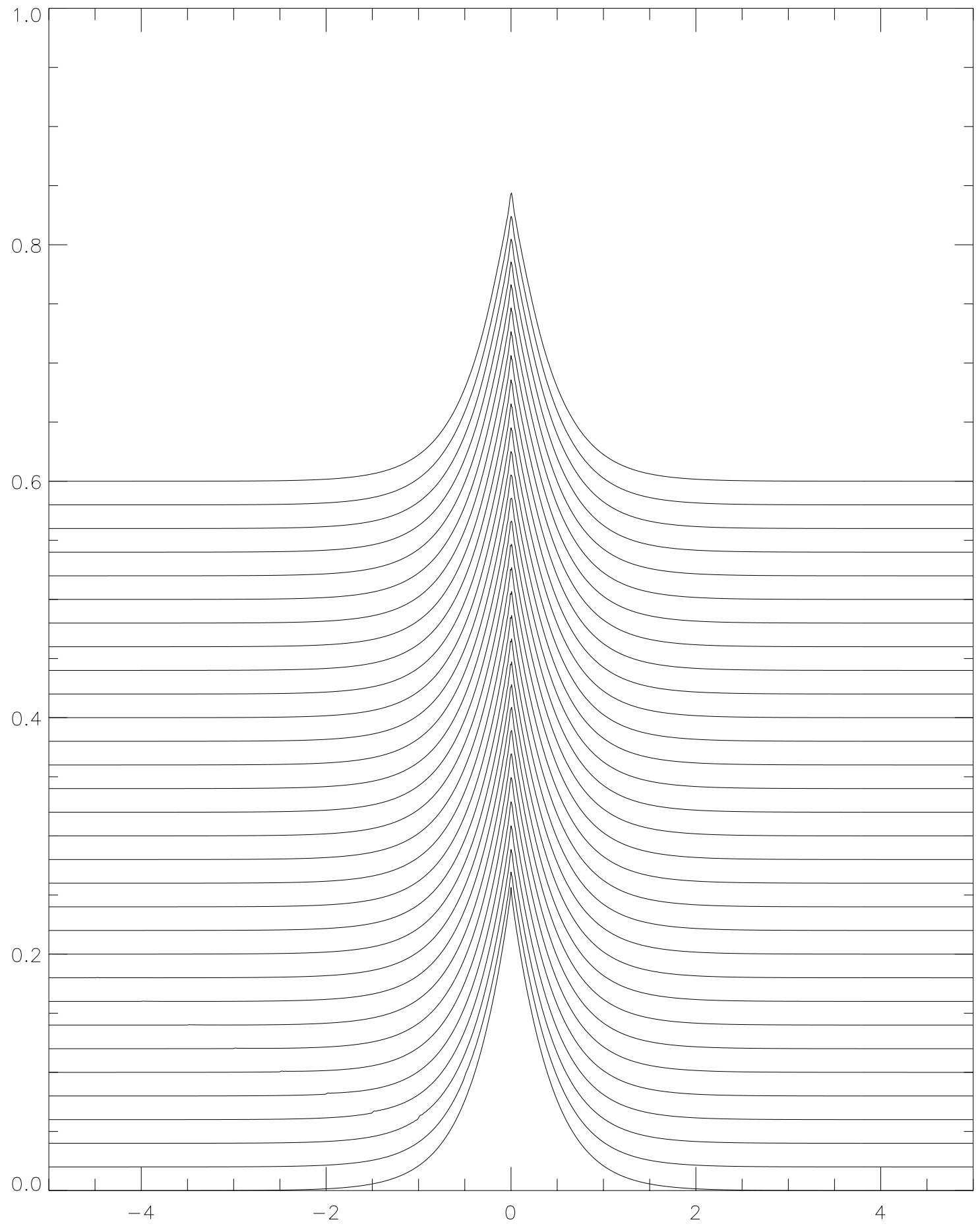

Figure 6: An example of a stable peakon. The plot shown is the field $\operatorname{Im} U_{1}$ vs. $x$ and $t$. The parameters are $\kappa=1.0, \alpha=1.95, \omega_{0}=1.5$, and $\omega=0.5$, with $\rho_{0}=0.04875$. 


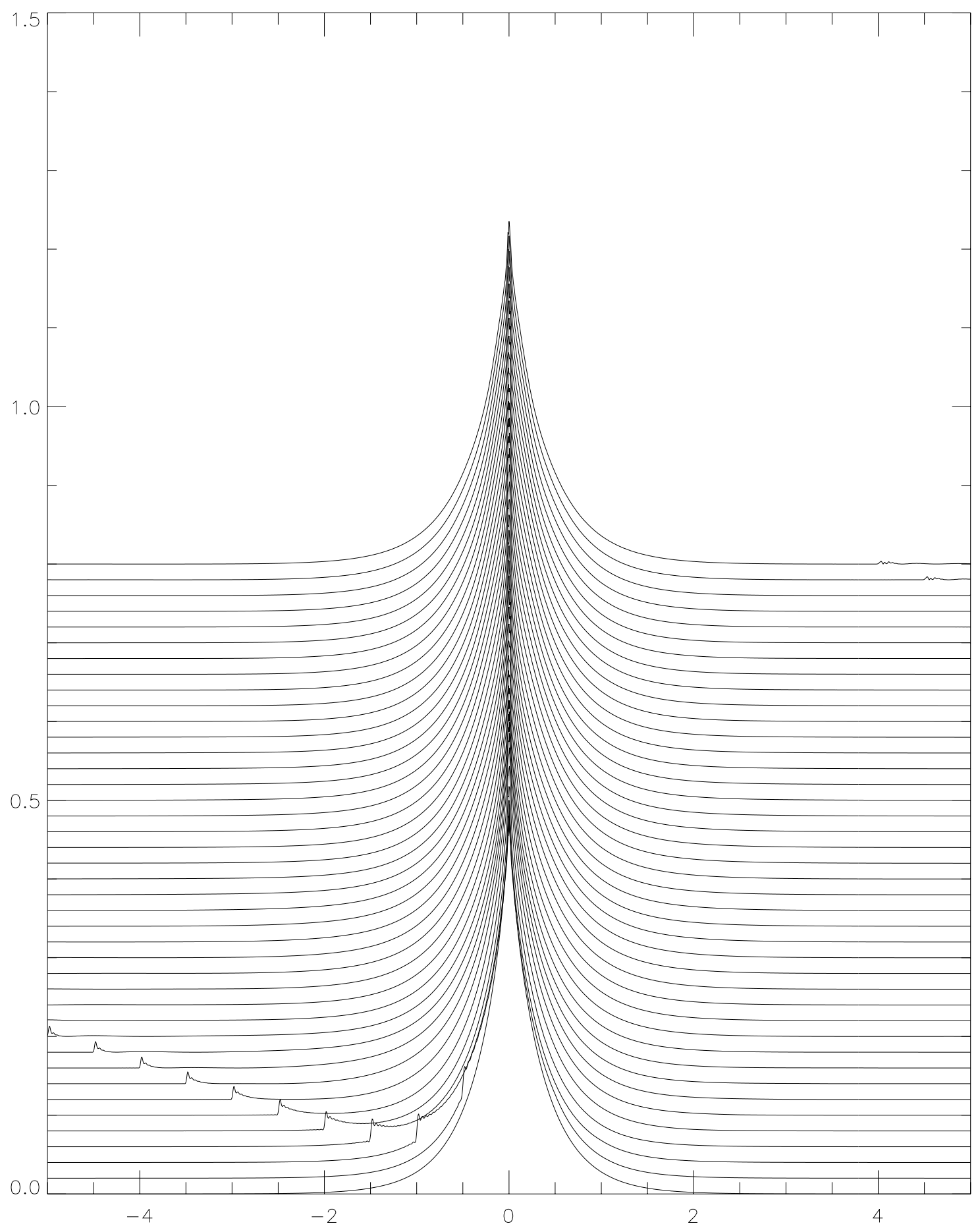

Figure 7: An example of a stable cuspon. The plot shown is the field $\operatorname{Im} U_{1}$ vs. $x$ and $t$. The parameters are $\kappa=1.0, \alpha=1.0, \omega_{0}=1.5$, and $\omega=0.5$, with $\rho_{0}=0.5$. 


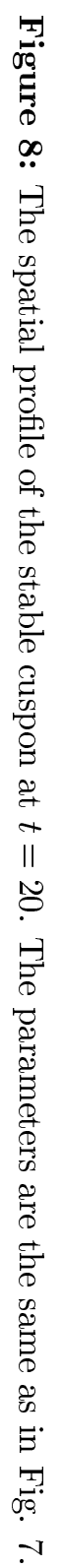

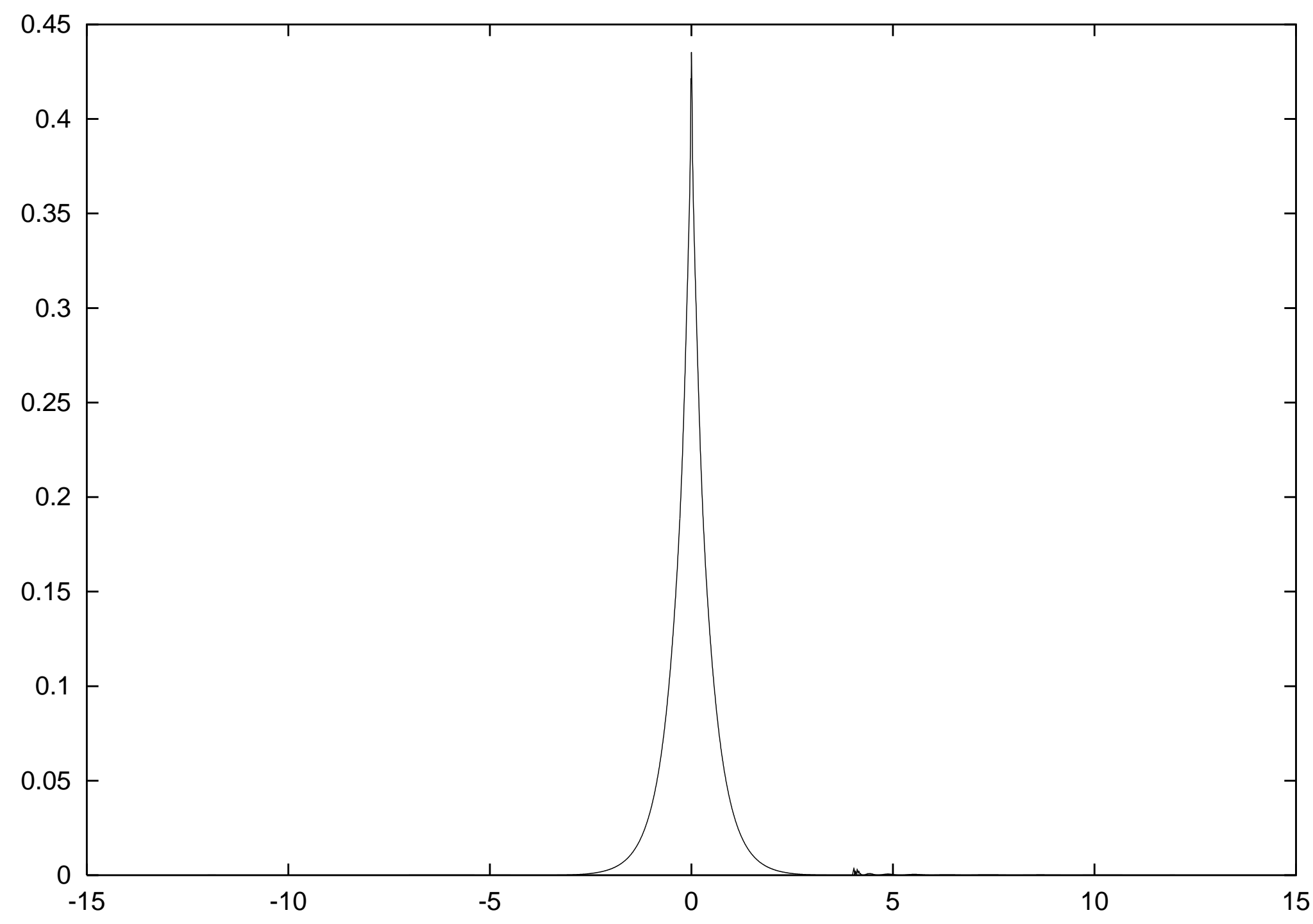




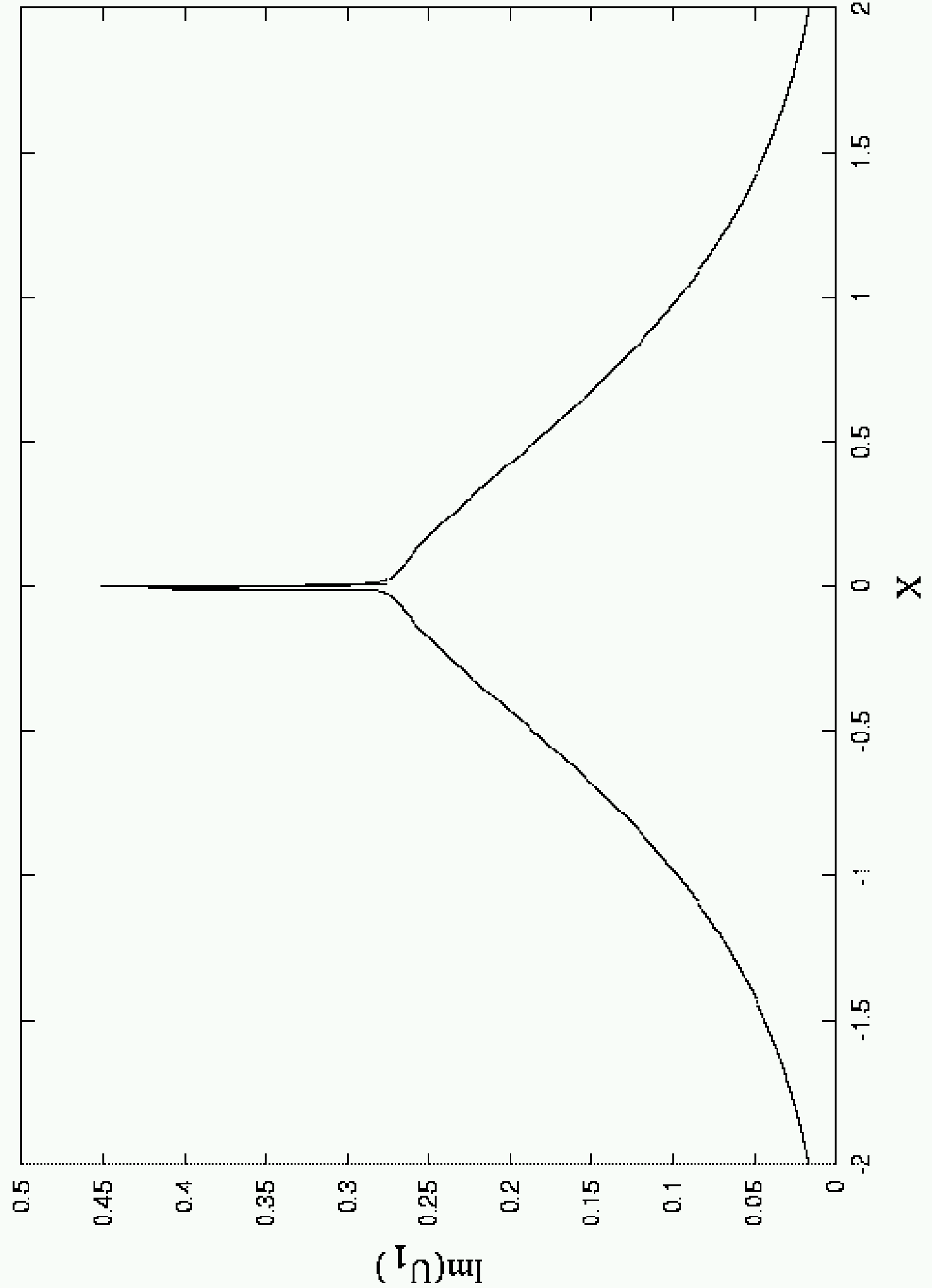

Figure 9: The spatial profile is shown for an unstable cuspon in terms of $\operatorname{Im} U_{1}$ at $t=10^{-3}$. The transition to collapse is clearly seen. The parameters are $\kappa=0.01, \alpha=1.1, \omega_{0}=0.1$, and $\omega=-0.3$, with $\rho_{0}=2.618$. 